\title{
Aspectos fonoaudiológicos e pediátricos na linguagem de gêmeos monozigóticos
}

\author{
Speech pathology and pediatric aspects in monozigotic twins language
}

Naraí Lopez Barbetta1', Ivone Panhoca², Maria de Lurdes Zanolli3

\section{RESUMO}

Objetivo: Observar o discurso familiar a respeito da gemelaridade, por meio de categorias inseridas nas práticas sociais, buscando subsidiar e delinear a contribuição do fonoaudiólogo e do pediatra no desenvolvimento de linguagem dos gêmeos monozigóticos.

Métodos: $\mathrm{O}$ trabalho apóia-se no método qualitativo de pesquisa e a análise dos dados foi realizada segundo a perspectiva histórico-cultural e o paradigma de natureza indiciária. Em 30 meses, foram realizadas oito entrevistas semi-estruturadas seqüenciais, iniciadas à revelação da gestação gemelar e continuadas em intervalos aproximados de três meses até cerca de dois anos de vida dos gêmeos, com dez famílias que tinham em sua composição direta um par de gêmeos idênticos.

Resultados: Sete categorias emergiram nos relatos obtidos com os familiares - a descoberta da gemelaridade, a escolha de nomes, o vestuário, a interação, a rotina, a linguagem e a identidade.

Conclusões: Os depoimentos, práticas, valores e pressupostos estão relacionados com o desenvolvimento destas crianças e apontam que essas famílias precisam de um acompanhamento diferenciado e específico, desde o momento gestacional.

Palavras-chave: gêmeos monozigóticos; desenvolvimento da linguagem; Pediatria; relações familiares; cuidados médicos.

\section{ABSTRACT}

Objective: To observe the way families express themselves about twins using categories related to social practices in order to help health care workers to contribute to the development of the speech of monozygotic twins.

Methods: The study uses the qualitative method, based on a historic-cultural perspective and the evidential paradigm. During 30 months, eight sequential semi-structured interviews were applied to ten families who had identical twins. The interviews began when the diagnosis of the twin gestation was announced to the families and continued at approximately three-month intervals until the twins were two years old.

Results: Seven categories were reported by the families during the interviews regarding the development of their twin children - announcement of the twin pregnancy, choice of names, choice of clothes, pattern of speech and identity of each child, as well as general routine and family interaction.

Conclusions: There is a relationship between the development of the language in monozygotic twins and the attitudes and values reported by their families. Families of twins may need specific and differentiated follow-up since the gestational period.

Key-words: twins monozygotic; language development; Pediatrics; family relations; medical care.
${ }^{1}$ Mestre e doutora em Saúde da Criança e do Adolescente pela Faculdade de Ciências Médicas da Universidade Estadual de Campinas (FCMUnicamp), professora do curso de Fonoaudiologia do Centro Universitário Nossa Senhora do Patrocínio (CEUNSP) e do Centro de Especialização em Fonoaudiologia Clínica, São Paulo, SP, Brasil

${ }^{2}$ Doutora em Ciências pelo Instituto de Estudos da Linguagem da Unicamp, professora da Faculdade de Fonoaudiologia da Pontifícia Universidade Católica de Campinas, professora visitante do programa de pós-graduação Saúde da Criança e do Adolescente da FCM-Unicamp, Campinas, SP, Brasil

${ }^{3}$ Doutora em Pediatria e professora do Departamento de Pediatria da FCMUnicamp, Campinas, SP, Brasil
Endereço para correspondência:

Naraí Lopez Barbetta

Rua Izabel Negrão Bertotti, 141 - apto. 81 - Mansões Santo Antônio CEP 13087-508 - Campinas/SP

E-mail: wnbarbetta@terra.com.br

Recebido em: 17/1/2008

Aprovado em: 18/4/2008 


\section{Introdução}

Estudos sobre gêmeos, especificamente sobre o desenvolvimento de sua linguagem, têm demonstrado o quanto este pode estar alterado em função de aspectos biológicos e interacionais. No que se refere aos aspectos biológicos, é fato que grande parte das crianças gêmeas apresenta intercorrências pré, peri e pós-natais. O desenvolvimento fetal de gêmeos ocorre num espaço menor devido à presença de mais um irmão no útero e vários estudos demonstram que eles podem nascer antes do que as crianças únicas, além de apresentarem baixo peso e estatura no nascimento ${ }^{(1)}$. Tais intercorrências são consideradas fatores de risco para possíveis alterações de linguagem ${ }^{(2,3)}$. Neste contexto, observa-se elevada taxa de mortalidade perinatal em gemelares, fortemente influenciada pelo baixo peso e prematuridade, devido à associação com desordens respiratórias, metabólicas, imunológicas e neurológicas. Soma-se aqui outro aspecto relevante que diz respeito aos índices de Apgar baixos entre os recém-nascidos gêmeos ${ }^{(4,5)}$.

Quanto aos aspectos interacionais, verifica-se que a estreita relação intragemelar pode reduzir a necessidade do desenvolvimento verbal e diminuir a motivação para se comunicar, levando ao aparecimento da chamada linguagem secreta, ou seja, uma comunicação própria entre os gêmeos ${ }^{(6-8)}$. Assim, tendo sempre a companhia do outro, não surgiria a necessidade objetiva de contato lingüístico com outras pessoas, constituindo um fator cristalizador do atraso. Eles seriam um par auto-suficiente ${ }^{(9)}$. Além disso, é descrito que as dificuldades de linguagem dos gêmeos podem decorrer do reduzido tempo de atenção e interação dispensado pela mãe, visto que duas crianças requerem muitos cuidados simultâneos ${ }^{(2)}$.

Quando se restringe tais particularidades aos gêmeos monozigóticos, verificam-se alguns fatores adicionais neste universo de intercorrências biológicas e interacionais. Durante a fase gestacional dos gêmeos monozigóticos, há ainda a competição por recursos intra-útero ${ }^{(10,11)}$. Gêmeos idênticos criados juntos também têm um ambiente mais semelhante, pois tendem a estudar na mesma escola e a partilhar mais atividades extracurriculares.

Assim, partindo-se da premissa que a família é o primeiro grupo no qual a criança está inserida e do qual começa a ser membro integrante e participativo, há que se considerar sua influência na constituição destes novos seres ${ }^{(12)}$. No caso específico de gêmeos monozigóticos, o nascimento de dois bebês tão parecidos pode desafiar os estoques interacional, relacional, de conhecimento e de afetividade do continente familiar que os recebe, visto que, numa gestação, esperase a chegada de apenas uma criança, isto é, a família está preparada para receber um único bebê e dar a ele toda a condição necessária para seu desenvolvimento dentro daquele contexto de grupo familiar ${ }^{(13)}$.

Algumas práticas podem acompanhar todo esse processo de concepção, nascimento e desenvolvimento dos gêmeos, principalmente quando idênticos. Inclui-se aqui o fato de usar roupas iguais ou com diferença apenas na cor, escolher nomes com semelhanças fonéticas, estabelecer rotinas parecidas quanto à alimentação e sono e manter atitudes similares para com as crianças. Estes hábitos, freqüentemente observados nas famílias de gêmeos, são, muitas vezes, reforçados nas relações sociais de vizinhança e amizade dos diferentes grupos sociais. Isso pode ocorrer a ponto de se configurar como um aspecto negativo, não havendo, muitas vezes, o respeito pela individualidade e pelo processo de constituição da identidade de cada membro do par, culminando em dificuldade posterior de aceitação pelo grupo social a que pertencem, caso desejem firmar a diferença como fator principal de sua individualidade ${ }^{(14-16)}$.

Dessa maneira, parece que a situação de desenvolvimento de gêmeos, quanto à identidade e linguagem, permeada por tais práticas sociais, pode caminhar para a formação de um estigma: o fato de duas crianças serem gêmeas monozigóticas está automaticamente vinculado à condição sine qua non de que a semelhança não é apenas física, mas envolve aspectos de identidade e de aceitação social.

A Pediatria é a especialidade médica dedicada ao cuidado da criança e do adolescente, por meio de uma assistência integral em todos os níveis de complexidade da atenção à saúde, priorizando a promoção à saúde e a prevenção de doenças, assim como o diagnóstico precoce e o tratamento oportuno ${ }^{(17)}$. O pediatra é o profissional que mantém estreita relação com a família dessas crianças desde o início das suas vidas, sendo de extrema importância sua participação no processo de orientação e/ou encaminhamento dessas famílias no que diz respeito a questões sobre o desenvolvimento de linguagem e a constituição de identidade das crianças gêmeas monozigóticas ${ }^{(18-20)}$.

Diante desse contexto, o objetivo deste estudo foi observar o discurso familiar a respeito da gemelaridade, por meio de categorias inseridas nas práticas sociais, buscando subsidiar e delinear a contribuição do fonoaudiólogo e do pediatra no desenvolvimento de linguagem dos gêmeos monozigóticos. 


\section{Métodos}

Este projeto de pesquisa foi aprovado pelo Comitê de Ética e Pesquisa da Faculdade de Ciências Médicas da Universidade Estadual de Campinas (FCM-Unicamp). O termo de consentimento livre e esclarecido foi assinado por todas as famílias participantes. $\mathrm{O}$ trabalho apóia-se no método qualitativo de pesquisa, que busca significado nas vivências trazidas por indivíduos acerca dos múltiplos fenômenos pertinentes a um problema ${ }^{(21)}$.

A coleta de dados ocorreu por entrevistas semi-estruturadas, realizadas pela pesquisadora responsável. Acredita-se que esta modalidade de entrevista possibilita ao entrevistado a oportunidade de discorrer sobre o tema proposto com certa liberdade e, ao pesquisador, fazer os redirecionamentos necessários para abordar suas hipóteses ou pressupostos ${ }^{(22,23)}$.

As entrevistas giraram em torno de depoimentos de pais e irmãos sobre as vivências familiares desde a revelação da gestação gemelar até o nascimento/desenvolvimento dos gêmeos. A pesquisadora iniciava com uma pergunta geral como: "Como estão as crianças?", e a partir da resposta dos familiares presentes, propunha temas (rotina das crianças, vestuário, interação, identificação individual, desenvolvimento, mudanças intrafamiliares, etc.) para serem explorados e manifestados no discurso familiar.

A primeira entrevista ocorreu no momento do diagnóstico da gestação gemelar monozigótica, a segunda logo após o nascimento (primeiro/segundo mês) e as demais ocorreram em intervalos aproximados de três meses. Este intervalo visou facilitar o acompanhamento do desenvolvimento das crianças em fases importantes do processo de aquisição de linguagem e da formação da identidade ${ }^{(24)}$. Dessa maneira, foram obtidas oito entrevistas com cada família, até aproximadamente o $26^{\circ}$ mês de vida.

Todas as entrevistas foram gravadas em vídeo na residência de cada uma das famílias, pois assim poderia se observar, de forma integral, a dinâmica da interação entre os sujeitos e registrar as falas dos interlocutores. Além da filmagem, as entrevistas foram transcritas ortograficamente para posterior seleção dos episódios a serem analisados.

Dados do Sistema de Informações de Nascidos Vivos ${ }^{(25)}$ mostraram que, em Campinas de um total de 13.659 nascimentos, 255 foram duplos. Considerando-se o caráter qualitativo da pesquisa, para compor o corpus, incluíram-se no estudo dez famílias, sendo as mesmas selecionadas a partir de registros de acompanhamento pré-natal de dois grandes hospitais da região de Campinas. Esse número de famílias foi determinado de acordo com o critério de saturação ${ }^{(26)}$.

Todas as famílias necessariamente tinham que ter, em sua composição direta, um par de gêmeos idênticos, sendo este o fator de inclusão determinante. Os demais fatores, tais como diferenças de idade, condição socioeconômica e cultural, local de domicílio, raça e crença religiosa favoreceram a heterogeneidade das famílias, o que foi considerado um aspecto positivo, por se aproximar da condição real de diversidade, presente em qualquer grupo social.

Como fator de exclusão, evitou-se a participação de famílias que tinham gêmeos fraternos ou que apresentaram algum fator de disruptura, tais como falecimento de um dos cônjuges, manifestações psicopáticas ou casos de depressão intensa em um dos membros da família, dependência química de qualquer natureza de qualquer integrante do grupo familiar. Entendeu-se que a presença de tais fatores desviaria o foco de atenção de todo o grupo familiar, o que não era desejável.

A perspectiva histórico-cultural propõe estudar o comportamento em mudança e suas condições sociais de produção, valorizando-se, assim, seu caráter histórico. Ressalta-se, também, o papel fundamental das relações sociais e concebe o estudo do homem, como um ser que se constitui imerso na cultura, nas experiências coletivas e práticas sociais e como produtor-intérprete de sua identidade ${ }^{(27)}$. Outra visão discute um paradigma de natureza indiciária como uma perspectiva que conduz à valorização de indícios de processos em andamento. O paradigma indiciário desdobra-se por meio de argumentos que apontam a importância dos pormenores, freqüentemente considerados negligenciáveis no estudo dos fenômenos. Aqui, decifrar e ler pistas é estabelecer conexões coerentes entre os eventos, e, assim sendo, o componente narrativo faz parte das interpretações indiciárias ${ }^{(28)}$.

\section{Resultados}

No que concerne à caracterização das famílias estudadas, todos os genitores freqüentaram apenas o ensino fundamental (completo ou não). Das dez famílias, oito residiam na periferia da cidade de Campinas, em moradias com pouca infra-estrutura, sem saneamento básico e fizeram o acompanhamento pré-natal e parto pelo Sistema Único de Saúde. Com relação à quantidade de filhos anteriores ao nascimento dos gêmeos, quatro famílias não tinham filhos e seis famílias tinham de um a dois filhos, com idades variando de cinco a 16 anos. 
Quadro 1 - síntese dos dados obtidos junto às famílias

\begin{tabular}{|c|c|c|c|}
\hline Indicadores de análise & Descoberta & Nomes & Vestuário \\
\hline $\begin{array}{l}\text { Panorama geral de todas as } \\
\text { famílias }\end{array}$ & $\begin{array}{l}\text { Todas as famílias relataram } \\
\text { o sentimento de surpresa, } \\
\text { choque e emoção muito forte. }\end{array}$ & $\begin{array}{l}\text { Sete famílias fizeram a opção } \\
\text { de nomes com semelhança } \\
\text { fonética (Renan e Renato), } \\
\text { iniciando com a mesma letra } \\
\text { (Laís e Larissa) e com a } \\
\text { mesma extensão (Marcelo e } \\
\text { Murilo). } \\
\text { Duas optaram por nomes } \\
\text { compostos. } \\
\text { Uma escolheu nomes } \\
\text { diferentes. }\end{array}$ & $\begin{array}{c}\text { Seis famílias escolheram usar } \\
\text { roupas iguais com diferença } \\
\text { apenas na cor e quatro delas } \\
\text { usavam sempre roupas } \\
\text { idênticas. }\end{array}$ \\
\hline Rotina & Interação & Linguagem & Identidade \\
\hline
\end{tabular}

Considerando o objetivo do trabalho, o período da coleta de dados (aproximadamente 30 meses com cada família), a quantidade de episódios e informações obtidas e o ambiente natural (residência de cada família) onde transcorreram as entrevistas, realizou-se uma análise mostrando que as seguintes categorias emergiram dos relatos obtidos: a descoberta da gemelaridade; a escolha de nomes; o vestuário; a interação; rotina; a linguagem; a identidade. Tais aspectos estão descritos no Quadro 1.

\section{Discussão}

Todas as famílias observadas relataram o momento de surpresa, choque e emoção muito forte quando da descoberta da gemelaridade. Isso reforça o quão inusitado é o fator semelhança e como o significado desse fato passa também a ser compartilhado culturalmente, organizando o grupo familiar em torno de representações e simbolismos daí advindos ${ }^{(29)}$.

Observou-se que, em sete famílias, o discurso intrafamiliar confirmou o desejo de escolher nomes com semelhanças fonéticas, iniciando com a mesma letra e com extensão equivalente, e das famílias restantes, duas optaram por nomes compostos e uma escolheu nomes diferentes. Nota-se, nesta categoria, a presença de um valor extremamente forte de escolher nomes que deveriam, a princípio, identificar as crianças como diferentes membros daquela família, já que os nomes funcionam como elos de filiação e de pertinência ${ }^{(30)}$. Mas, com a opção por nomes tão parecidos, provoca-se justamente um efeito contrário, algo que parecia passar despercebido pelas famílias. O prenome de cada um dos gêmeos os igualava, dificultando a identificação individual. Por isso, alguns dos 
pais acabavam instituindo apelidos, numa tentativa de favorecer a identificação, principalmente pelos familiares diretos. Esse relato dos pais parece se relacionar com a dificuldade em assimilar a presença de gêmeos no seio da família, que acabam sendo "dois vistos como um".

Já com relação ao vestuário, a opção de usar roupas iguais com diferença apenas na cor esteve presente em seis famílias e as demais usavam, sempre, roupas idênticas para ambos os filhos gêmeos. Mais uma vez observa-se, da parte dos pais, a tendência de olhar as duas crianças sob o prisma de uma. Roupas similares ou idênticas acabam por igualar mais uma vez estas crianças, que já contam com o fator da semelhança física tão marcante $\mathrm{e}^{(29,30)}$.

O estabelecimento de rotina idêntica para as crianças foi observado em todas as famílias, quanto aos aspectos de alimentação, horários de repouso/sono, de passeio e de brincadeira. Sabe-se, também, que uma rotina constante e regular favorece a formação de conceitos temporais e espaciais, no curso do desenvolvimento da criança. No caso dos gêmeos idênticos, essa constatação, em relação à rotina das crianças, vem, novamente, reforçar o pressuposto de uma igualdade entre eles, desconsiderando-se o fato de serem duas pessoas, com especificidades que deveriam ser observadas e respeitadas ${ }^{(13)}$.

No aspecto da interação, foi importante a fala das famílias quanto ao bom contato intragemelar em todas as fases do desenvolvimento. Todas as famílias, em algum momento das entrevistas, relataram as semelhanças no processo de aprendizagem de habilidades das crianças. Neste aspecto, as crianças apresentaram, segundo os pais, dias de diferença entre um dos gêmeos aprender algo e seu irmão conseguir realizar a mesma atividade, da mesma forma. Tem-se, aqui, a expectativa familiar apoiada em pressupostos sobre os gêmeos idênticos, que reforçam as práticas cotidianas quanto à semelhança no período de novas aquisições ${ }^{(13,24,30)}$.

Em relação à linguagem, em cinco famílias, as crianças apresentaram, até o término da pesquisa, o desenvolvimento de linguagem diferenciado para a idade, pois um dos gêmeos era mais eficiente na oralidade, sendo muitas vezes o porta-voz da dupla, segundo a observação dos pais. Dentre essas famílias, duas relataram a presença de uma linguagem diferente entre as crianças (por volta de 12 meses de idade $)^{(7)}$. Já em duas famílias, de acordo com os pais, os gêmeos apresentaram um atraso no desenvolvimento de linguagem, mostrando uma comunicação oral ainda pobre, com vocábulos ininteligíveis e só utilizando a fala quando estritamente necessária ${ }^{(2)}$. Em outra família, devido à prematuridade, questão sempre evidente na fala da mãe, as crianças apresentaram um atraso significativo no desenvolvimento de linguagem. Os depoimentos de duas famílias não destacaram problemas de linguagem com os gêmeos. Uma possível explicação para estas diferenciações pode ser o aspecto das contingências sociais diferenciadas interferindo no desenvolvimento de linguagem dos gêmeos monozigóticos ${ }^{(2,10,15)}$.

Quanto à identificação/identidade, os dados revelaram que principalmente os familiares indiretos e pessoas amigas tiveram dificuldade para identificar os membros do par. Em uma família, a mãe considerou a hipótese da interferência do nome parecido nesta confusão e recorreu ao uso de apelido para um dos gêmeos. Em outra, a mãe optou por usar uma pulseira em uma das crianças para facilitar o reconhecimento e a identificação ${ }^{(15)}$. Lembrando que a construção da identidade é estreitamente relacionada às práticas discursivo-sociais, bem como à construção das relações sociais entre os falantes e à dos sistemas de conhecimento e crenças, observou-se que os hábitos e valores do grupo social aqui pesquisado apareceram com grande força, acabando por contribuir para a formação de estigma com relação a essas crianças que, no dia a dia, não eram reconhecidas (ou diferenciadas) pelo nome, aparência física, vestimenta e por seus desejos ou anseios ${ }^{(15,17,30)}$. Dessa forma, a possibilidade de identificação dos gêmeos dependia, na maioria das vezes, da palavra da mãe (em cinco famílias, os familiares recorriam à mãe para saber qual o nome de cada membro do par) ou das ações da mãe (em três famílias, a mãe estipulou apelidos ou um acessório para identificar as crianças frente aos familiares).

Considerando que os primeiros anos de vida da criança são fundamentais para a formação de sua identidade, construção de sua linguagem e sua constituição como indivíduo, pôde-se observar o quanto a situação de gemelaridade pode interferir nessa dinâmica de desenvolvimento ${ }^{(15)}$.

A cultura inclui um conjunto de atitudes, crenças, idéias, visões de mundo e códigos de comportamento para um determinado grupo social, inserido num certo período histórico ${ }^{(31)}$. Assim, no contexto social e cultural das famílias aqui enfocadas, consideradas as condições econômicas e educacionais, os aspectos analisados, da forma como se mostraram, revelaram-se como os mais corretos para os padrões de criação de filhos, quando eles são gêmeos.

Pode-se concluir que, frente à semelhança de gêmeos monozigóticos, as categorias enfocadas mostraram-se fortes e determinantes, sobrepondo-se às eventuais preocupações das famílias em relação ao desenvolvimento da linguagem e da 
identidade dessas crianças. Os depoimentos, práticas, valores e pressupostos observados neste trabalho indicam que tais famílias precisam de um acompanhamento diferenciado e específico, desde o momento gestacional. Cabe aos profissio- nais da saúde, principalmente ao pediatra e ao fonoaudiólogo, oferecerem um pronto acesso a programas de orientação e apoio sobre o desenvolvimento de gêmeos, visando promover a saúde das crianças e a prevenção de possíveis alterações.

\section{Referências bibliográficas}

1. Beiguelman B, Colleto GMDD, Franchi-Pinto $\mathrm{C}$, Krieger $\mathrm{H}$. Birth weight of twins: 2. Fetal genetic effect on birth weight. Genet Mol Biol 1998;21:155-8.

2. Mogford K. Desenvolvimento de linguagem em gêmeos. In: Bishop D, Mogford $\mathrm{K}$, editores. Desenvolvimento da linguagem em circunstâncias excepcionais. Rio de Janeiro: Revinter; 2002. p. 99-122.

3. Schirmer CR, Portuguez MW, Nunes NL. Clinical assessment of language development in children at age 3 years that were born preterm. Arq Neuropsiquiatr 2006;64:926-31.

4. Beiguelman $B$, Franchi-Pinto $C$. Perinatal mortality among twins and singletons in a city in southeastern Brazil, 1984-1996. Genet Mol Biol 2000;23(1):15-23.

5. Franchi-Pinto C, Colletto GMDD, Krieger H, Beiguelman B. Genetic effect on Apgar score. Genet Mol Biol 1999;22:13-6.

6. Bishop DV, Bishop SJ. "Twin language": a risk factor for language impairment? J Speech Lang Hear Res 1998;41:150-60.

7. Bakker P. Autonomous languages of twins. Acta Genet Med Gemellol (Roma) 1987;36:233-8.

8. Barbetta NL, Panhoca I. Gêmeos idênticos no grupo terapêutico fonoaudiológico: a construção da linguagem e da subjetividade. Pró-Fono Rev Atual Cient 2003;15:139-48.

9. Luria AR, Yudovich FI. Linguagem e desenvolvimento intelectual na criança. $2^{\mathrm{a}}$ ed. Porto Alegre: Artes Médicas; 1987.

10. Mogford-Bevan K. Developmental language impairments with complex origins: learning from twins and multiple birth children. Folia Phoniatr Logop 2000;52:74-82

11. Beiguelman B. [book on the Internet]. O estudo de gêmeos [cited $2007 \mathrm{Sep}$ 28]. Available from: http://www.desvirtual.com/bbeiguel/GEMEOS.PRN.pdf

12. Weber DE, Vares MA, Mota HB, Keske-Soares M. Desenvolvimento do sistema fonológico de gêmeos monozigóticos com desvio fonológico: correlação a fatores genéticos e ambientais. Rev CEFAC 2007;9:32-9

13. Arruda DC, Marcon SS. A família em expansão: experienciando intercorrências na gestação e no parto do bebê prematuro com muito baixo peso. Texto Contexto Enferm 2007;16:120-8.

14. Rusch N, Angermeyer MC, Corrigan PW. Mental illness stigma: concepts, consequences and initiatives to reduce stigma. Eur Psychiatry 2005;20:529-39.
15. Molon SI. Subjetividade e constituição do sujeito em Vygotsky. Petrópolis: Vozes; 2003.

16. Goffman E. Estigma. Rio de Janeiro: Guanabara Koogan; 1988.

17. Pessoa JHL. O exercício da pediatria nos dias atuais. Rev Paul Pediatr 2004;22:188-9.

18. Rabelo BGR, Salomão LM, Carivali PA, Leite ICG. Algumas considerações sobre o grau de conhecimento dos pediatras sobre questões fonoaudiológicas. Fono Atual 2004;27:4-10.

19. Schirmer CR, Fontoura DR, Nunes ML. Distúrbios da aquisição da linguagem e da aprendizagem. J Pediatr (Rio J) 2004;80(Suppl 2):S95-103.

20. Zocoli AMF, Riechel FC, Zeigelboim BS, Marques JM. Hearing: a pediatricians aproach. Rev Bras Otorrinolaringol (Engl Ed) 2006;72:617-23.

21. Turato ER. Métodos qualitativos e quantitativos na área da saúde: definições, diferenças e seus objetos de pesquisa. Rev Saude Publica 2005;39:507-14

22. Martins HHTS. Metodologia qualitativa de pesquisa. Educ Pesq (USP) 2004;30:289-300.

23. Wengraf T. Qualitative research interviewing: semi-structured, biographical and narrative methods. London: Sage Publications; 2004.

24. Mendes DMLF, Moura MLS. Desenvolvimento da brincadeira e linguagem em bebês de 20 meses. Psic Teor e Pesq (Brasília) 2004;20:215-22.

25. Campinas - Secretaria da Saúde [homepage on the Internet]. Dados de nascidos vivos de mães residentes em Campinas, SP; 2002 [cited 2008 Apr 2]. Available from: http://antigo.campinas.sp.gov.br/saude/

26. Minayo MCS. Desafio do Conhecimento: pesquisa qualitativa em saúde. $9^{a}$ ed. São Paulo: Hucitec; 2006.

27. Vygotsky LS. A formação social da mente. São Paulo: Martins Fontes; 1989.

28. Ginzburg C. Mitos, emblemas, sinais - morfologia e história. $2^{a}$ ed. São Paulo: Companhia das Letras; 2003.

29. Pino A. As marcas do humano: às origens da constituição cultural da criança na perspectiva de Lev S. Vigotski. São Paulo: Cortez; 2005.

30. Cerveny CMO, Rabinovich EP. Família e genealogia. In: Cerveny CMO, editor. Família e genealogia. São Paulo: Casa do Psicólogo; 2006. p. 97-114.

31. Ginzburg C. O queijo e os vermes. $9^{\mathrm{a}}$ ed. São Paulo: Companhia das Letras 2000. 